\title{
Educação permanente com agentes comunitários de saúde: dificuldades para identificar, acolher e cuidar de usuários com transtornos de saúde mental
}

\author{
Continuing education with community health agents: difficulties in identifying, welcoming and \\ caring for users with mental health disorders \\ Formación continuada con agentes de salud comunitarios: dificultades para identificar, acoger $\mathbf{y}$ \\ atender a usuarios con trastornos de salud mental
}

\author{
Marcelo Xavier da Silva Sousa \\ ORCID: https://orcid.org/0000-0002-0040-5986 \\ Faculdade Santo Agostinho, Brasil \\ E-mail: marcelo_x21@hotmail.com \\ Ana Letícia Portela Moura Oliveira \\ ORCID: https://orcid.org/0000-0003-3356-5836 \\ Hospital São Marcos, Brasil \\ E-mail: analeticiapmoliveira@ hotmail.com \\ Alana Ilmara Pereira da Costa \\ ORCID: https://orcid.org/0000-0001-9909-1835 \\ Fundação Municipal de Saúde de Teresina, Brasil \\ E-mail: alana_ilmara@ hotmail.com \\ Maria da Cruz Oliveira Ferreira Moura \\ ORCID: https://orcid.org/0000-0003-0173-4500 \\ Universidade Federal do Piauí, Brasil \\ E-mail: mdc_of@hotmail.com \\ Lívia Maria Nunes de Almeida \\ ORCID: https://orcid.org/0000-0002-5857-4635 \\ Universidade Federal do Piauí, Brasil \\ E-mail: livialmeida@ufpi.edu.br \\ Natália Pereira Marinelli \\ ORCID: https://orcid.org/0000-0003-4696-3518 \\ Universidade Federal do Piauí, Brasil \\ E-mail: nataliamarinelli@ufpi.edu.br
}

\begin{abstract}
Resumo
A saúde mental não está dissociada da saúde geral. Diante do crescimento do número de usuários com transtorno de saúde mental associado à dificuldade de identificação precoce desse público pela equipe de saúde, de adesão do paciente ao tratamento, de corresponsabilização da família e de abertura da sociedade para o acolhimento desses usuários, destaca-se a necessidade de intervenções nessa área, como qualificação do processo de cuidado através da implementação de educação permanente. Trata- se de um estudo exploratório e descritivo, de caráter qualitativo, tipo relato de experiência sobre a elaboração de um plano operativo. Esse trabalho tem como objetivo qualificar os Agentes Comunitários de Saúde (ACS's) sobre a identificação, o acolhimento e o cuidado de usuários com transtornos de saúde mental no município de Duque Bacelar- MA. Esse projeto promoverá educação permanente com ACS's, trabalhando as dificuldades para identificar, acolher e cuidar de usuários com transtornos de saúde mental. Portanto, favorecerá a ampliação dos atos de escuta, observação e percepção dos ACS diante das demandas que apresentam problemas mentais, além do desenvolvimento de habilidades e a conscientização da necessidade de atitudes.
\end{abstract}

Palavras-chave: Transtorno mental; Educação permanente; Agentes comunitários de saúde.

\begin{abstract}
Mental health is not dissociated from general health. Given the growth in the number of users with mental health disorders associated with the difficulty of early identification of this public by the health team, patient adherence to treatment, co-responsibility of the family and society's openness to receive these users, there is a need for interventions in this area, such as qualification of the care process through the implementation of continuing education. This is an exploratory and descriptive study, of qualitative nature, type of experience report on the
\end{abstract}


development of an operative plan. This work aims to qualify the Community Health Agents (CHAs) on the identification, reception and care of users with mental health disorders in the municipality of Duque Bacelar - MA. This project will promote permanent education with CHWs, working on the difficulties in identifying, welcoming and caring for users with mental health disorders. Therefore, it will favor the amplification of the listening, observation and perception acts of the CHWs in face of the demands that present mental problems, besides the development of skills and the awareness of the need for attitudes.

Keywords: Mental disorders; Continuing education; Community health workers.

\section{Resumen}

La salud mental no está disociada de la salud general. Ante el creciente número de usuarios con trastornos de salud mental, asociado a la dificultad de identificación precoz de este público por parte del equipo sanitario, la adherencia del paciente al tratamiento, la corresponsabilidad de la familia y la apertura de la sociedad a la acogida de estos usuarios, destaca la necesidad de intervenciones en este ámbito, como la cualificación del proceso asistencial a través de la implantación de la formación continuada. Se trata de un estudio exploratorio y descriptivo, de carácter cualitativo, tipo informe de experiencia sobre el desarrollo de un plan operativo. Este trabajo tiene como objetivo capacitar a los Agentes Comunitarios de Salud (ACS) en la identificación, recepción y atención de usuarios con trastornos de salud mental en el municipio de Duque Bacelar - MA. Este proyecto promoverá la formación continua con los TSC, trabajando en las dificultades para identificar, acoger y atender a los usuarios con trastornos de salud mental. Por lo tanto, favorecerá la ampliación de los actos de escucha, observación y percepción de los CHAs ante las demandas que presentan problemas mentales, además del desarrollo de habilidades y la toma de conciencia de la necesidad de actitudes.

Palabras clave: Trastornos mentales; Formación continua; Trabajadores sanitarios de la comunidad.

\section{Introdução}

A saúde mental não está dissociada da saúde geral. E por isso faz-se necessário reconhecer que as demandas de saúde mental estão presentes em diversas queixas relatadas pelos pacientes que chegam aos serviços de Saúde, em especial da Atenção Básica. As práticas em saúde mental na Atenção Básica podem e devem ser realizadas por todos os profissionais de Saúde. O que unifica o objetivo dos profissionais para o cuidado em saúde mental devem ser o entendimento do território e a relação de vínculo da equipe de Saúde com os usuários, mais do que a escolha entre uma das diferentes compreensões sobre a saúde mental que uma equipe venha a se identificar (Brasil, 2019).

Diante do crescimento do número de usuários com transtorno de saúde mental associado à dificuldade de identificação precoce desse público pela equipe de saúde, de adesão do paciente ao tratamento, de corresponsabilização da família e de abertura da sociedade para o acolhimento desses usuários, destaca-se a urgente necessidade de intervenções nessa área, através da implementação de ações de governabilidade do NASF e das ESF do município de Duque Bacelar, como qualificação do processo de cuidado através da implementação de educação permanente.

Nesse sentido, Brasil afirma que a consolidação e o aprimoramento da atenção básica como importante reorientadora do modelo de atenção à saúde no Brasil requerem um saber e um fazer em educação permanente que sejam encarnados na prática concreta dos serviços de saúde. A educação permanente deve ser constitutiva, portanto, da qualificação das práticas de cuidado, gestão e participação popular. Além da sua evidente dimensão pedagógica, deve ser encarada também como uma importante "estratégia de gestão", com grande potencial provocador de mudanças no cotidiano dos serviços, em sua micropolítica, bastante próximo dos efeitos concretos das práticas de saúde na vida dos usuários (Brasil, 2012).

O presente projeto elencou os ACS's como profissionais centrais, devido suas características profissionais de estarem em contato permanente com as famílias, desenvolvendo ações educativas, visando à promoção da saúde, à prevenção das doenças, representando a primeira forma de contato entre a comunidade e os serviços de saúde.

Diante do exposto, foi estabelecido como objetivo desse trabalho: qualificar os Agentes Comunitários de Saúde (ACS's) sobre a identificação, o acolhimento e o cuidado de usuários com transtornos de saúde mental no município de Duque 
Bacelar- MA.

\section{Metodologia}

Trata-se de um estudo exploratório e descritivo, de caráter qualitativo, tipo relato de experiência sobre a elaboração de um plano operativo.

A abordagem qualitativa auxilia na realização diagnósticos, detalhadamente, sobre um determinado problema social gerando propostas eficazes de como resolve-los, podendo ser utilizada nos processos de implementação ou mudanças organizacionais e institucionais. (Ludke \& André, 2013)

Yin (2001) evidencia a importância da construção de um plano de pesquisa até chegar o relatório final e Chiavenato (2004), afirma que antes de realizar funções como organização, direção, controle e coordenação é preciso planejar, pois ele representa a primeira e mais importante função administrativa.

O plano representa o produto de todo processo de planejamento. Serve para detalhar o processo de mudança e a situação desejada, e documentar os objetivos gerais e específicos, as operações, as ações e as estratégias necessárias para o alcance das metas traçadas. Para que o plano se mantenha atual, ele deve ser constantemente revisado e sempre que necessário, deve ocorrer a proposição de estratégias que o tornem adequado às novas situações (Tancredi, Barrios, Ferreira, 2002). O Planejamento Estratégico Situacional, a partir de seus fundamentos e métodos, propõe o desenvolvimento do planejamento como um processo participativo. Sendo assim, possibilita a incorporação dos pontos de vista dos vários setores sociais, incluindo a população, e que os diferentes atores sociais explicitem suas demandas, propostas e estratégias de solução, numa perspectiva de negociação dos diversos interesses em jogo. Essa participação enriquece o processo de planejamento, criando corresponsabilidade dos atores com a efetivação do plano de ação, dando mais legitimidade e, mesmo, viabilidade política ao plano (Campos, Faria \& Santos, 2010).

Segundo Pereira et al. (2018), toda estratégia que estimule o público-alvo trabalhar de modo ativo pode se tornar interessante nos processos educacionais. Entre elas, podemos destacar: o emprego da pedagogia por projetos e o uso da pedagogia por problemas por envolver os participantes ativamente na identificação de problemas e busca de soluções; o uso da pedagogia por problemas - os problemas ou busca pela resolução deles, de modo semelhante aos projetos podem fazer com que os alunos estudem ativamente.

Esse projeto, foi construído baseando-se nos quatros momentos que caracterizam o processo de planejamento estratégico situacional identificado por Matus: Explicativo, Normativo, Estratégico e Tático-Operacional. Cada momento representa uma visão dinâmica do processo de planejamento e se caracteriza pela permanente e constante interação e retomada de suas fases durante o processo de planejamento. ${ }^{5}$

Momento Explicativo: busca conhecer a situação atual, procurando identificar, priorizar e analisar seus problemas;

1. Momento Normativo: momento de elaboração de propostas de solução;

2. Momento Estratégico: busca analisar e construir viabilidade para as propostas de solução elaboradas, formulando estratégias para se alcançarem os objetivos traçados;

3. Momento Tático Operacional: momento de execução do plano (Campos; Faria; Santos, 2010).

Ainda para subsidiar a construção deste plano operativo, foram utilizados dados de bases como: BVS, SCIELO e Ministério da Saúde. 


\section{Resultados e Discussão}

\subsection{Revisão da Literatura}

A atual política de saúde mental brasileira é resultado da mobilização de usuários, familiares e trabalhadores da Saúde iniciada na década de 1980 com o objetivo de mudar a realidade dos manicômios. O movimento foi impulsionado pela importância que o tema dos direitos humanos adquiriu no combate à ditadura militar e alimentou-se das experiências exitosas de países europeus na substituição de um modelo de saúde mental baseado no hospital psiquiátrico por um modelo de serviços comunitários com forte inserção territorial. Nas últimas décadas, esse processo de mudança se expressa especialmente por meio do Movimento Social da Luta Antimanicomial e de um projeto coletivamente produzido de mudança do modelo de atenção e de gestão do cuidado: a Reforma Psiquiátrica (Brasil, 2013).

No Sistema Único de Saúde (SUS), a porta de entrada preferencial de todo usuário é a Atenção Primária. Ela deve ser capaz de realizar promoção e proteção da saúde, prevenção de agravos, diagnóstico, tratamento, redução de danos e manutenção da saúde, observando os princípios que regemo SUS, como a universalidade, integralidade e equidade. Logo, o acolhimento e cuidado prestado devemser destinados a todos indivíduos e coletividades levassem distinção de necessidades, crenças, valorese problemas de saúde.

Nesse sentido, a Saúde Mental e Atenção Básica são campos que convergem a um objeto comum e o que está em jogo em ambos é a superação das limitações da visão dualista do homem, a construção de um novo modelo dinâmico, complexo e não reducionista e a orientação para novas formasde prática na área de Saúde (Brasil, 2013).

Para Brasil, a Atenção Primária à Saúde compreende quatro atributos essenciais: o acesso (primeiro contato do indivíduo com o sistema de saúde), a continuidade do cuidado, a integralidade da atenção e a coordenação do cuidado dentro do sistema. Ademais, a presença de outras três características, chamadas atributos derivados, qualificam as ações em Atenção Primária à Saúde: a atenção à saúde centrada na família (orientação familiar), a orientação comunitária e a competência cultural (Brasil, 2014).

Para apoiar a Estratégia de Saúde da Família, ampliando sua abrangência de serviços, sua resolutividade e sua integralidade na oferta de ações nas redes, o Ministério da Saúde criou os Núcleos de Apoio à Saúde da Família (NASF), mediante a Portaria GM n 154, de 24 de janeiro de 2008, republicada em 4 de março de 2008.

O NASF é uma estratégia inovadora que tem por objetivo apoiar, ampliar, aperfeiçoar a atençãoe a gestão da saúde na Atenção Básica/Saúde da Família. Seus requisitos são, além do conhecimento técnico, o desenvolvimento de habilidades relacionadas ao paradigma da Saúde da Família. Deve estar comprometido, também, com a promoção de mudanças na atitude e na atuação dos profissionais da ESF e entre sua própria equipe (NASF), incluindo na atuação ações intersetoriais e interdisciplinares, promoção, prevenção, reabilitação da saúde e cura, além de humanização de serviços, educação permanente, promoção da integralidade e da organização territorial dos serviços de saúde (Brasil, 2014).

Assim, destaca-se um dos objetivos do NASF essencial na qualificação da atenção à saúde: os processos de educação permanente em saúde (EPS) nos territórios. Para esse processo ser resolutivo, precisa ser pautada no diagnóstico situacional de cada território, centrada na reflexão dos problemas de cada comunidade para que se atinja a transformação de realidades

A educação permanente não se trata de "espaços de capacitação", onde quem sabe ensina a quem não sabe, mas de encontros para reflexão das práticas a partir do compartilhamento de saberes de todos os participantes. As novas informações devem ser apresentadas de forma que mobilizem os trabalhadores a repensarem suas práticas para qualificarem 
suas ofertas de cuidado aos usuários, por isso é muito importante prestar atenção no modo como se fala, a fim de criar um ambiente respeitoso, inclusivo e positivo (Brasil, 2017).

A educação permanente deve embasar-se num processo pedagógico que contemple desde a aquisição/atualização de conhecimentos e habilidades até o aprendizado que parte dos problemas e desafios enfrentados no processo de trabalho, envolvendo práticas que possam ser definidas por múltiplos fatores (conhecimento, valores, relações de poder, planejamento e organização do trabalho etc.) e que considerem elementos que façam sentido para os atores envolvidos, gerando uma aprendizagem significativa (Brasil, 2012).

Freire (2008) afirma que o papel do facilitador é promover a curiosidade e a criticidade; reconhecer que o processo educacional é inacabado; respeitar a autonomia do especializando; mostrar responsabilidade, tolerância e bom senso; integrar intenção e gesto, comprometendo-se com a educação como forma de intervenção no mundo e de transformação da realidade.

É notória a singularidade nacional e internacional do Sistema Único de Saúde - SUS, assim como é notória a necessidade emergente de melhoria na aplicação dos serviços de saúde e políticas públicas desse sistema. Esse processo de melhoria perpassa por ações de microgestão, mesogestão e macrogestão da clínica aplicada. No entanto, o trabalhador de saúde está habituado a apontar apenas a mesogestão e a macrogestão como capazes de solucionar problemas e modificar os serviços de saúde. Os profissionais estão acomodados no pensamento de que não podem mudar o SUS no país e esquecem que vivem o SUS em seus cotidianos e tendo competência para interferir ativamente nele.

Outra ferramenta de grande relevância abordada entre ESF e NASF, é o Projeto Singular Terapêutico ou Matriciamento. Pinto et al. (2012), afirma que essa ferramenta amplia o potencial resolutivo perante os casos clínicos de saúde mental devido ao compartilhamento de informações territoriais, demandas clínicas e de procedimentos. Além de trabalhar a corresponsabilização entre equipe, usuário e família. O diferencial deste dispositivo terapêutico se pauta no compartilhamento de problemas vividos e na pactuação integrada das resoluções.

Pode-se transformar a prática da educação em saúde em uma ferramenta potente de aprendizagem para profissionais da saúde e usuários. Acrescenta-se ainda que discorrer sobre o processo saúde-doença-educação implica também em lidar com questões bastante subjetivas como o autocuidado do ser humano e responsabilização de cada um com o processo de saúde (pacientes, familiares e equipe de saúde). É importante destacar que usuários com transtornos mentais não representam usuários com deficiência mental, portanto esses usuários devem ser inseridos no processo-saúde-doença-educação sendo corresponsabilizados também.

Nesse sentido, o autocuidado consciente, libertador, autônomo, responsável, ativo, transformador se apresenta como um novo caminho a ser percorrido e a ser destacado dentro do processo de trabalho de profissionais que atuam na educação, na assistência social, entre outros ambientes, e, claro, em especial a área da saúde, por este, ser um lugar promotor de conflitos pessoais (Freire, 2013).

Trabalhar essa demanda traz consigo dificuldades de várias naturezas: dificuldade de acesso do usuário à unidade de saúde, falta de conhecimento do profissional, a fragmentação do paciente que passa a ser estigmatizado pelo problema mental, tendo suas outras necessidades clínicas negligenciadas, presença de mitos e preconceitos relacionados ao público com transtornos mentais, o que gera um acolhimento inadequado a essa demanda.

Tendo o ACS como primeira forma de acesso da população aos serviços de saúde, é necessário iniciar com essa categoria profissional o desenvolvimento de educação permanente visando efetividade das ações em saúde mental desde a etapa de primeiro contato desse usuário com a ESF. 
O Programa de Agentes Comunitários de Saúde (PACS) teve início no fim da década de 80. Foi criado pelo Ministério da Saúde em 1991 para buscar alternativas para melhorar as condições de saúde de suas comunidades. Trata-se de uma categoria de trabalhadores formada por membros própriacomunidade para garantir e melhorar o acesso da mesma aos serviços de saúde. Logo, o agente comunitário de saúde tem um papel muito importante no acolhimento, pois é membro da equipe que faz parte da comunidade, o que permite a criação de vínculos mais facilmente, propiciando o contato direto com a equipe.

É atribuição dos ACS, desenvolver atividades de promoção da saúde, de prevenção das doenças e agravos e de vigilância à saúde, por meio de visitas domiciliares e de ações educativas individuais e coletivas nos domicílios e na comunidade; e estar em contato permanente com as famílias, desenvolvendo ações educativas, visando à promoção da saúde, à prevenção das doenças eao acompanhamento das pessoas com problemas de saúde (Brasil, 2012).

A vinculação dos processos de educação permanente à estratégia de apoio institucional pode potencializar enormemente o desenvolvimento de competências de gestão e de cuidado na atenção básica, na medida em que aumenta as alternativas para o enfrentamento das dificuldades vivenciadas pelos trabalhadores em seu cotidiano (Brasil, 2012).

\subsection{Projeto Operativo}

Para Soeiro et al (2017), o Projeto Aplicativo constitui uma atividade curricular multidisciplinar com foco na construção de uma intervenção na realidade por meio da construção de um projetoaplicativo. Contempla a seleção, pactuação e caracterização de um problema para a construção de uma proposta de intervenção.

Esse projeto foi construído baseando-se nos quatros momentos que caracterizam o processo de planejamento estratégico situacional identificado por Matus (1993).

O Planejamento Estratégico constrói a partir das observações dos profissionais envolvidos na prestação do cuidado. "Esse delineamento se processa continuamente, renovando a compreensão de necessidades de saúde, de práticas profissionais e de organização do trabalho que evoluem, emtransformação mútua e permanente, em direção a uma noção de qualidade na atenção à saúde pautada pela compreensão desse processo de mudança contínua” (Freire, 2013).

\subsection{Primeira Etapa: Momento Explicativo}

Segundo dados do Senso Demográfico do Instituto Brasileiro de Geografia e Estatística (IBGE), Duque Bacelar conta com uma população estimada de 11.208 habitantes (IBGE, 2017).

De acordo com a "Enciclopédia dos Municípios Brasileiros" volume XV, de autoria do IBGE, a cidade de Duque Bacelar, deu-se exclusivamente a interesse particulares de duas famílias de destaque no município de Coelho Neto. O clã dos "Bacelar" cuja geração é considerada autóctone, era de maior influência do município. Vindo do Alto Sertão Maranhense, o capitalista José de Ribamar Oliveira com sua família, estabeleceu-se no povoado "Garapa", durante 15 anos, sem maiores problemas com a família Bacelar. Com a morte do chefe do clã, o coronel Raimundo de Melo Bacelar, em 1954, começouo desentendimento entre as duas famílias o que veio originar a criação do município de Duque Bacelar, pelo desmembro, os separativistas, visto contar com a influência política e o prestígio de um dos membros que na época era Deputado Estadual. O topônimo "Duque Bacelar", foi dado ao novo município em homenagem póstuma ao coronel Raimundo Melo Bacelar, conhecido pela alcunha "Duque Bacelar". O município foi criado pela Lei Estadual n 1294, de 7 dezembro de 1954, contudo o povoado "Garapa" sede do município foi elevado à categoria de sede em $1^{\circ}$ de janeiro de 1955 (IBGE, 2008). 
De acordo com o Centro Nacional de Estabelecimentos de Saúde, em relação a Saúde, omunicípio disponibiliza dos seguintes estabelecimentos:

- Serviço de Atendimento Móvel de Urgência (SAMU);

- Unidade de Vigilância Sanitária;

- Secretaria Municipal de Saúde;

- Centro de Atendimento e Diagnóstico;

- Hospital Presidente Médice;

- Unidade de Vigilância Epidemiológica (FUNASA);

- 03 Unidades de Saúde da Família;

- Polo de Academia da Saúde Francisco Pereira da Silva;

- Centro de Reabilitação de Duque Bacelar;

- 02 Postos de Saúde.

Mesmo com uma estrutura propícia para o acolhimento e cuidado aos usuários, foram observadas, durante reuniões com os ACS das equipes de ESF, atendimentos domiciliares compartilhados e solicitações para atendimento individual, a existência de dificuldades relacionadas a esse processo de trabalho, principalmente, quando vinculado ao usuário com transtornos mentais.

O município não apresenta Centro de Apoio Psicossocial (CAPS), tendo que encaminhar a demanda para o município vizinho (Coelho Neto- MA), o que dificultada um pouco mais a assistência a esses usuários. No entanto, grande parte da demanda encaminhada não necessita, no momento, de atendimento especializado. A ESF deve ser a ordenadora do cuidado ao usuário com transtorno mental, devido seus atributos de universalidade, integralidade, territorialização e regionalização dos processos de saúde.

Tendo os ACS como primeira forma de acesso da população aos serviços de saúde, evidenciamos nessa fase, as dificuldades dos Agentes Comunitários de Saúde para identificar, acolher e cuidar de usuários com transtornos de saúde mental no município de Duque Bacelar.

Um estudo de Delors (2000) propôs quatro eixos do conhecimento e da formação continuada, considerados norteadores: aprender a conhecer; aprender a fazer; aprender a conviver; e aprender a ser. Esses eixos sintetizam a necessidade profissional diante da atenção em saúde mental. Cada um precisa de conhecimento científico sobre esse tema, fazer um acolhimento integral a esse público, conviver com esse público e sensibilizar a comunidade da importância da não marginalização doconvívio social desses usuário, e por, precisa-se de humanização na atenção a saúde em todos os aspecto.

\subsection{Segunda Etapa: Momento Normativo}

Considerando a proposta geral desse projeto de qualificar os Agentes Comunitários de Saúde (ACS's) sobre a identificação, o acolhimento e o cuidado de usuários com transtornos de saúde mental no município de Duque Bacelar- MA, foram elencadas as seguintes propostas de solução:

- Implementar a Educação Permanente dos ACS’s sobre Saúde Mental;

- Identificar os usuários com transtorno de saúde mental de cada ESF;

- Realizar Projeto Terapêutico Singular dos usuários com transtorno de saúde mental e respectivas famílias; 
Nessa fase, foram elaboradas as seguintes propostas: Implementar a Educação Continuada dos ACS's sobre Saúde Mental; Identificar os usuários com transtorno de saúde mental de cada ESF e Realizar Projeto Terapêutico Singular dos usuários com transtorno de saúde mental e respectivasfamílias.

\subsection{Terceira Etapa: Momento Estratégico}

Nessa fase, foram traçadas estratégias para cada proposta solução estabelecida anteriormente,elencado os responsáveis para execução de cada uma. (Quadro 1)

Quadro 1. Estratégias E Responsáveis Traçados Para Cada Proposta Solução Estabelecida. Teresina (P), Brasil, 2021

\begin{tabular}{|c|c|}
\hline ESTRATÉGIAS & RESPONSÁVEIS \\
\hline \multicolumn{2}{|c|}{ Proposta solução: Implementar a Educação Continuada dos ACS's sobre Saúde Mental; } \\
\hline $\begin{array}{l}\text { Realizar o processo de educação permanente em Saúde Mental com osACS’s. } \\
\text { O treinamento será realizado em } 2 \text { módulos com encontros presenciais a cadamês. }\end{array}$ & Psicólogo do NASF \\
\hline \multicolumn{2}{|c|}{ Proposta solução: Identificar os usuários com transtorno de saúde mental de cada ESF } \\
\hline $\begin{array}{l}\text { Solicitar das ESF planilha de identificação dos usuários com transtorno desaúde } \\
\text { mental, bem como atualização mensal das mesmas. }\end{array}$ & $\begin{array}{l}\text { Enfermeira; } \\
\text { Médico(a);ACS. }\end{array}$ \\
\hline \multicolumn{2}{|c|}{$\begin{array}{l}\text { Proposta solução: Realizar Projeto Terapêutico Singular dos usuários com transtorno de saúde } \\
\text { mental e respectivas famílias; }\end{array}$} \\
\hline $\begin{array}{l}\text { Estabelecer o Projeto Terapêutico Singular dos usuários com transtorno de saúde mental e } \\
\text { respectivas famílias: será programado o dia do acolhimento dousuário e familiares na } \\
\text { UBS. }\end{array}$ & $\begin{array}{c}\text { NASF: Psicólogo, Nutricionista, } \\
\text { Fisioterapeuta, Fonoaudióloga } \\
\text { ESF: Enfermeira, Médico(a), } \\
\text { Dentista, Técnicos de } \\
\text { Enfermagem e Saúde Bucal, } \\
\text { ACS. }\end{array}$ \\
\hline
\end{tabular}

Fonte: Autores.

Em suma, a integração das três etapas anteriores consolida a elaboração do plano Operativo desse estudo (Quadro 2). 
Quadro 2. Plano operativo. Teresina (PI), Brasil, 2021.

\begin{tabular}{|c|c|c|c|c|}
\hline $\begin{array}{c}\text { Situação } \\
\text { Problema }\end{array}$ & Objetivos & $\begin{array}{l}\text { Metas/ } \\
\text { Prazos }\end{array}$ & Ações/ Estratégias & Responsáveis \\
\hline \multirow[t]{2}{*}{$\begin{array}{l}\text { Dificuldades dos } \\
\quad \text { ACS's } \\
\text { para identificar, } \\
\text { acolher e } \\
\text { cuidar de } \\
\text { usuários com } \\
\text { transtornos de } \\
\text { saúde mental }\end{array}$} & $\begin{array}{l}\text { Implementar a } \\
\text { Educação } \\
\text { Continuada dos } \\
\qquad \begin{array}{c}\text { ACS's } \\
\text { sobre Saúde } \\
\text { Mental; }\end{array}\end{array}$ & 30 dias & $\begin{array}{l}\text { Realizar o processo de educação permanente em Saúde } \\
\text { Mental com os ACS's. } \\
\text { O treinamento será realizado em } 2 \text { módulos com encontros } \\
\text { presenciais acada mês. } \\
\text { Os conteúdos abordados terão como base o Caderno da } \\
\text { Atenção Básica de Saúde Mental No } 34 \text { do Ministério da } \\
\text { Saúde. Brasília-DF, } 2013 \text {. } \\
1^{\circ} \text { módulo será abordado: } \\
\text { Cuidado da Pessoa em Sofrimento: }\end{array}$ & Psicólogo do NASF \\
\hline & & & $\begin{array}{l}\text { Conceitos gerais e aplicações práticas. } \\
\text { 1.1 O cuidado em saúde mental: como é o seu trabalho na } \\
\text { Atenção Básica } \\
\text { 1.2 A Política Nacional de Atenção Básica } \\
\text { 1.3 Núcleos de Apoio à Saúde da Família } \\
\text { 1.4 Política Nacional de Saúde Mental } \\
\text { 1.5 E o que pode ser entendido como uma intervenção em } \\
\text { saúde mental? } \\
\text { 1.6 Ações terapêuticas comuns aos profissionais da } \\
\text { Atenção Básica } \\
\text { 1.7 O cuidado que dá certo em saúde mental } \\
2 \text { A definição de cuidado, sofrimento, pessoa. } \\
\text { 3. A construção da Rede de Cuidados Compartilhados } \\
\text { 4. Os projetos terapêuticos singulares. No } 2^{\circ} \text { módulo } \\
\text { será abordado: } \\
\text { 1 Transtornos Mentais mais comum em Duque Bacelar- } \\
\text { MA: } \\
\text { 1.1 Depressão } \\
\text { 1.2 Transtorno de Ansiedade (Trans do Pânico e TAG) } \\
\text { 1.3 Transtorno Bipolar } \\
\text { 1.4 Esquizofrenia } \\
\text { 1.5 TDAH } \\
2 \text { Características básicas para detectar transtorno mental. } \\
3 \text { Uso abusivo x racional de medicamentos } \\
\text { psicofarmacológicos. } \\
4 \text { A importância do ACS na prevenção e controle das } \\
\text { pessoas com transtorno Mental. }\end{array}$ & \\
\hline & $\begin{array}{l}\text { Identificar os } \\
\text { usuários com } \\
\text { transtorno de } \\
\text { saúde mental de } \\
\text { cada ESF }\end{array}$ & 60 dias & $\begin{array}{l}\text { Solicitar das ESF planilha de identificação dos usuários } \\
\text { com transtorno de saúde mental, bem como atualização } \\
\text { mensal das mesmas. }\end{array}$ & $\begin{array}{l}\text { Enfermeira; } \\
\text { Médico(a); ACS. }\end{array}$ \\
\hline
\end{tabular}




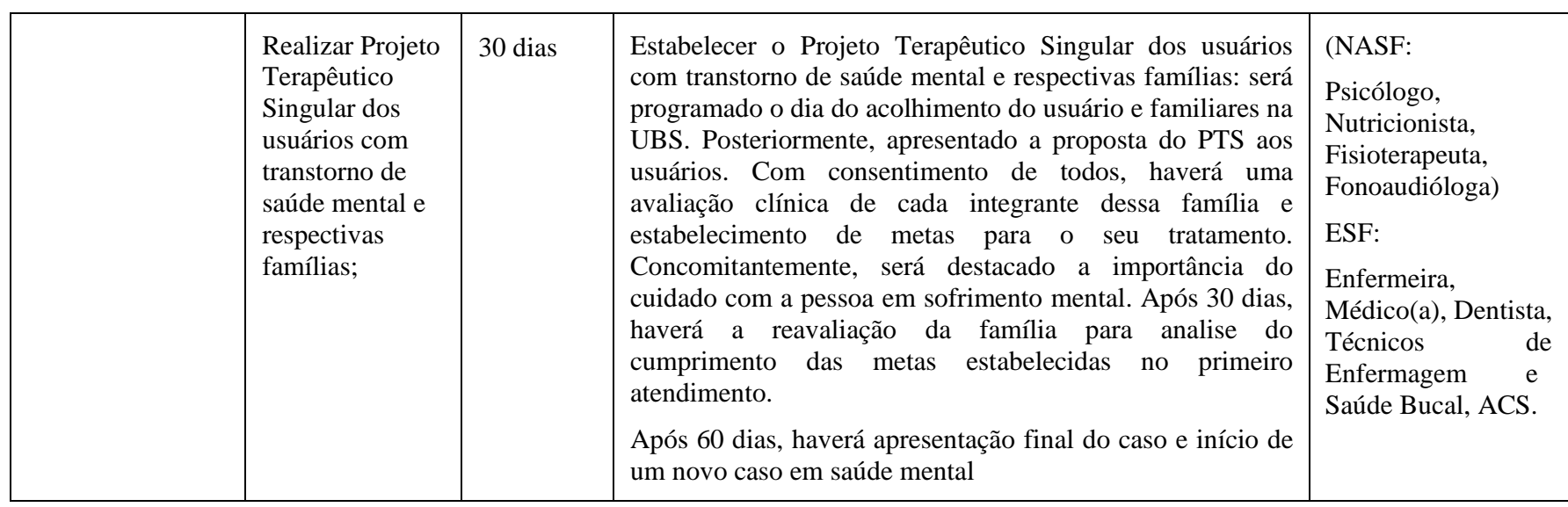

Fonte: Autores.

\subsection{Quarta Etapa: Momento Tático Operacional}

A execução do plano iniciará em Janeiro de 2018 com os agentes comunitários de saúde das 04 Estratégias Saúde da Família do Município de Duque Bacelar. Os encontros acontecerão separadamente por ESF e ocorrerão em 02 (dois) módulos distintos.

No decorrer da história, o processo educacional apresentou mudanças essenciais, através dos quais se estabeleceu uma nova abordagem na relação entre educador e educando. Neste novo cenário da sociedade contemporânea, o educando deve assumir o seu papel na construção do conhecimento e o educador atuar como facilitador desse conhecimento (Farias, Martin \& Cristo, 2015). Com isso, cabe destacar, que a educação como hoje existe, é resultado de experiências ao longo da história, das diferentes demandas da sociedade, pois, através da experiência, é possível se reconstruir valores, desenvolver novos critérios de estrutura da sociedade e assim, acrescentar novos valores (Bevilaqua, 2014).

\section{Conclusão}

As práticas em saúde mental na atenção primária em saúde devem ser fortalecidas por todos os profissionais de saúde, uma vez que a APS representa o centro ordenador das redes de saúde, de menor densidade tecnológica, mas a base do desencadeamento do processo saúde-doença em uma determinada população.

Esse projeto favorecerá a ampliação dos atos de escuta, observação e percepção dos ACS diante das demandas que apresentam problemas mentais. Favorecerá ainda, o desenvolvimento de habilidades e a conscientização da necessidade de atitudes. Buscará tornar inquestionável como o comprometimento profissional, as habilidades no processo de trabalho, a padronização do cuidado e o empoderamento de responsabilidades e condutas é capaz de produzir melhorias no cuidado prestado a cada grupo social, principalmente esse que ainda é tão estigmatizado pela sociedade.

Por isso, a formação do profissional que atua no SUS encontra destaque por refletir diretamente na qualidade das intervenções de saúde prestadas baseadas na realidade vivida pelo usuário. Para trabalhar a saúde precisa- se entender as bases do processo saúde-doença, levar em consideração o perfil demográfico da população de abrangência, fatores biológicos, socioeconômico, o perfil das condições agudas e crônicas da doença, o valor epidemiológico das doenças transmissíveis ainda em alta no Brasil, sem excluir a cultura, valores, desejos e interesses das pessoas.

Indubitavelmente, destaca-se a necessidade de mais estudos publicadas em bibliotecas científicas oficiais e aprofundamento no tema em pesquisa futuras, abordando as consequências de pesquisas baseadas em projetos aplicativos 
abrangendo saúde mental, uma vez que, trabalhar com usuários de transtornos mentais traz consigo dificuldades de várias naturezas: dificuldade de acesso do usuário à unidade de saúde, falta de conhecimento do profissional, a fragmentação do paciente que passa a ser estigmatizado pelo problema mental, tendo suas outras necessidades clínicas negligenciadas, presença de mitos e preconceitos relacionados ao público com transtornos mentais, o que gera um acolhimento inadequado a essa demanda.

\section{Referências}

Bevilaqua, A. P. (2014). John Dewey e a Escola Nova no Brasil. Ciência \& Luta de Classes Digital, Ano I, v.1, n. 1.

Brasil (2012). Ministério da Saúde. Política Nacional de Atenção Básica. Brasília: Ministério da Saúde.

Brasil (2013). Ministério da Saúde. Secretaria de Atenção à Saúde. Departamento de Atenção Básica. Saúde mental. Brasília: Ministério da Saúde.

Brasil. (2014). Ministério da Saúde. Núcleo de Apoio à Saúde da Família. v. 1. Brasília: Ministério da Saúde.

Brasil. (2017). Ministério da Saúde. Ministério da Saúde. Secretaria de Atenção à Saúde. Departamento de Atenção Básica. Contribuições dos Núcleos de Apoio à Saúde da Família para a Atenção Nutricional. Brasília: Ministério da Saúde.

Campos, F. C. C., Faria, H. P. \& Santos, M. A. (2010). Planejamento e avaliação das ações em saúde. 2. ed. Belo Horizonte: Universidade Federal de Minas Gerais.

Chiavenato, I. (2004). Administração nos novos tempos. 2. ed. Rio de Janeiro: Elsevier, 610p.

Delors, J. (2000). Educação: um tesouro a descobrir. Relatório para a Unesco da Comissão Internacional sobre Educação para o Século XXI. 4. ed. São Paulo/Brasília (DF): Cortez/Unesco.

Farias, P. A. M., Martin, A, L. A. R. \& Cristo, C. S. (2015). Aprendizagem Ativa na Educação em Saúde: Percurso Histórico e Aplicações. Revista Brasileira de Educação Médica, Rio de Janeiro, v. 39, n. 1, p. 143-150.

Freire, P. (2008). Pedagogia da autonomia: saberes necessários à prática educativa. 3. ed. São Paulo: Paz e Terra.

Freire, P. (2013). Educação como prática da liberdade. 15 edição. Rio de Janeiro: Paz e Terra.

IBGE. (2008). Instituto Brasileiro de Geografia e Estatística, 2008. Maranhão, Duque Bacelar, Histórico.

IBGE. (2017). Instituto Brasileiro de Geografia e Estatística. Maranhão, Duque Bacelar.

Ludke, M. \& Andre, M. E . D. A. (2013). Pesquisas em educação: uma abordagem qualitativa. São Paulo: E.P.U.

Matus, C. (1993). Política, planejamento e governo. Brasília, DF: Instituto de Economia Aplicada.

Pereira, A. S. et al. (2018). Metodologia da pesquisa científica. 1. ed. - Santa Maria, RS: UFSM, NTE.

Pinto, A. G. A. (2012). Apoio matricial como dispositivo do cuidado em saúde mental na atenção primária: olhares múltiplos e dispositivos para resolubilidade. Ciência \& Saúde Coletiva, 17(3):653-660.

Soeiro, E. (2017). Curso de especialização em gestão da Clínica nas regiões de saúde: caderno do curso 2017. São Paulo: Hospital Sírio Libanês.

Tancredi, F. B., Barrios S. R. L. \& Ferreira, J. H. G. (2002). Planejamento em saúde. São Paulo: FSP- USP.

Yin, R. K. (2015). O estudo de caso. Porto Alegre: Bookman. 